Proceedings of the International School and Conference on Optics and Optical Materials, ISCOM07, Belgrade, Serbia, September 3-7, 2007

\title{
Gaussian-Induced Rotation in Triangular Photonic Lattices
}

\author{
D. Jović*, S. Prvanović, R. Jovanović and M. Petrović
}

Institute of Physics, Pregrevica 118, 11080 Belgrade, Serbia

Time-dependent rotation of counterpropagating mutually incoherent self-trapped Gaussian beams in periodic optically induced fixed photonic lattices is numerically investigated. Rotation occurs for some values of control parameters. For parameters of such rotation, the solitonic solutions are found using modified Petviashvili's method. It is shown that they correspond to the lowest values of propagation constant in the power diagrams and relation between observed rotation and less confined discrete solitonic solutions are demonstrated.

PACS numbers: 42.65.Tg, 42.65.Sf

\section{Introduction}

Light propagation in periodic photonic structures has attracted growing interest to both fundamental physics and applications in recent years [1]. Optically induced photonic lattices (PLs) provide an excellent opportunity for studying many interesting phenomena of light propagation balanced between discreteness and nonlinearity $[2-5]$. Some of them are the intrinsic localized modes, so-called discrete or lattice solitons [6-10]. Another interesting phenomenon attractive for all-optical manipulation of light is soliton rotation, theoretically considered [11] and experimentally confirmed [12] in the Bessel-like ring lattices. In addition, soliton rotation can be found in other diverse fields, such as Bose-Einstein condensates, but also in radially periodic potentials (lattices) [13]. We numerically investigated vortex-induced rotating structures [14], but in periodic PLs. Here, we demonstrate Gaussian-induced rotation in these PLs and discuss its relation with less confined discrete solitons. We report a numerically time-dependent stable rotating beam structure, resulting from the collision of two counterpropagating (CP) mutually incoherent self-trapped Gaussian beams in periodic optically induced fixed PL. Bifurcation from Gaussian into rotating structures is due to the

*corresponding author; e-mail: jovic@phy.bg.ac.yu 
fact that a spatial symmetry breaking is associated with a supercritical Hopf bifurcation in the time domain. In these systems the rotation dynamics is realized through tunneling between lattice sites $[15,16]$. For parameters for which rotational dynamics occurs, we have analyzed solitonic solutions by using the modified Petviashvili method [17, 18], and we have found the relation between rotating structures and solitonic solutions. Quite unexpectedly, it has been discovered that solitonic solutions, derived for the steady state of governing equations, can help in finding parameters of Gaussian-induced time-dependent rotation. All of this is done by considering hexagonal/triangular PL with a central defect.

\section{Numerical results and discussion}

The behavior of CP beams in PLs is described by a time-dependent model consisting of wave equations in the paraxial approximation for the propagation of $\mathrm{CP}$ beams and a relaxation equation for the generation of the space charge field in the photorefractive crystal. The model equations in the computational space are [19]:

$$
\begin{aligned}
& \mathrm{i} \partial_{z} F=-\Delta F+\Gamma E F, \quad-\mathrm{i} \partial_{z} B=-\Delta B+\Gamma E B, \\
& \tau \partial_{t} E+E=-\frac{I+I_{\mathrm{g}}}{1+I+I_{\mathrm{g}}},
\end{aligned}
$$

where $F$ and $B$ are the envelopes of the forward and backward propagating beams. $\Delta$ is the transverse Laplacian, $\Gamma$ is the beam coupling constant, and $E$ - the homogeneous part of the space charge field. $I=|F|^{2}+|B|^{2}$ is the laser light intensity, measured in units of the background intensity $I_{\mathrm{d}}$. $I_{\mathrm{g}}$ is the intensity distribution of the optically induced lattice array. $\tau$ is the relaxation time of the crystal, which also depends on the total intensity, $\tau=\tau_{0} /\left(1+I+I_{\mathrm{g}}\right)$, and consequently on the spatial coordinates. The numerical procedure is as in Ref. [19].

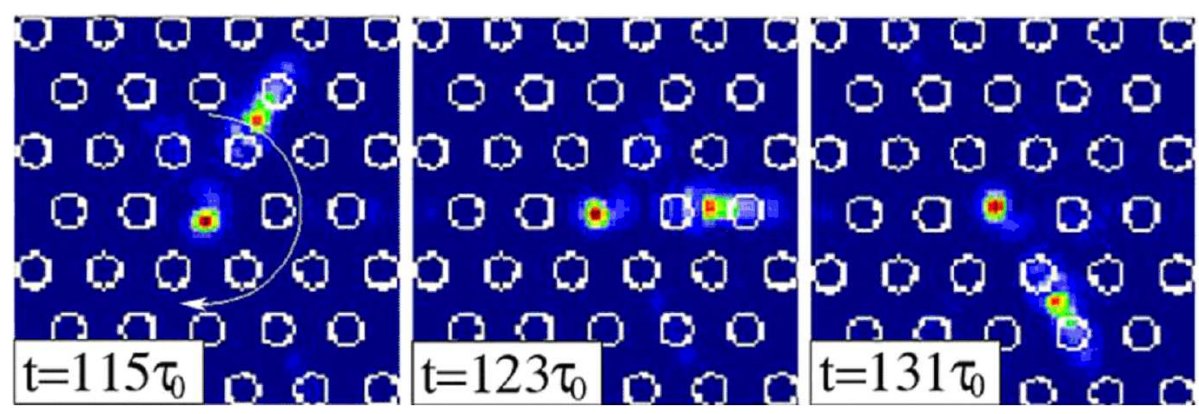

Fig. 1. Gaussian-induced rotation: intensity distribution of backward beam at its exit face of the crystal, presented at different times. Parameters $\Gamma=25$, input FWHM of CP Gaussian beams $11 \mu \mathrm{m}$, beam power 3.80, the propagation distance is $L=2 L_{\mathrm{D}}=8 \mathrm{~mm}$ and $\left|F_{0}\right|^{2}=\left|B_{L}\right|^{2}=1 I_{\mathrm{d}}$. 
We consider a lattice array with a triangular arrangement of beams and with the central beam absent. We launch head-on CP Gaussian beams into the center of the lattice, parallel to the lattice beams. We found very clear and periodical rotation (Figs. 1 and 2) in a very narrow region of the control parameters. Each Gaussian beam collapses to a displaced soliton-like beam, and after transient dynamics they start to rotate indefinitely. Since for parameters of such stable periodic solutions there are no stable steady states and since, in numerics, Eq. (2) becomes equivalent to the scalar nonlinear delay differential equation, this phenomenon is recognized as supercritical Hopf bifurcation [20]. The central parts of Gaussians rotate regularly in the center of the lattice, owing to the defect, along the whole crystal. Filaments rotate with the constant period away from the center along symmetry axes of the lattice by tunneling between lattice sites, but only close to its exit face of the crystal. Gaussian-induced rotating structures present soliton-like solutions, because they preserve shape along the main symmetry direction during rotation. The physical origin beyond rotation is incoherent interaction and spontaneous symmetry breaking, while rotation is realized through Zener tunneling $[15,16]$. Observed rotating structures are stable in the presence of up to $5 \%$ noise added to the input beam intensity and phase. Spontaneous symmetry breaking via noise chooses the direction of rotation, both directions occur with $50 \%$ probability. For the same control parameters, CP Gaussian beams show very irregular dynamical behavior in the absence of any lattice, but very stable propagation is found in the lattices without defects (not shown).
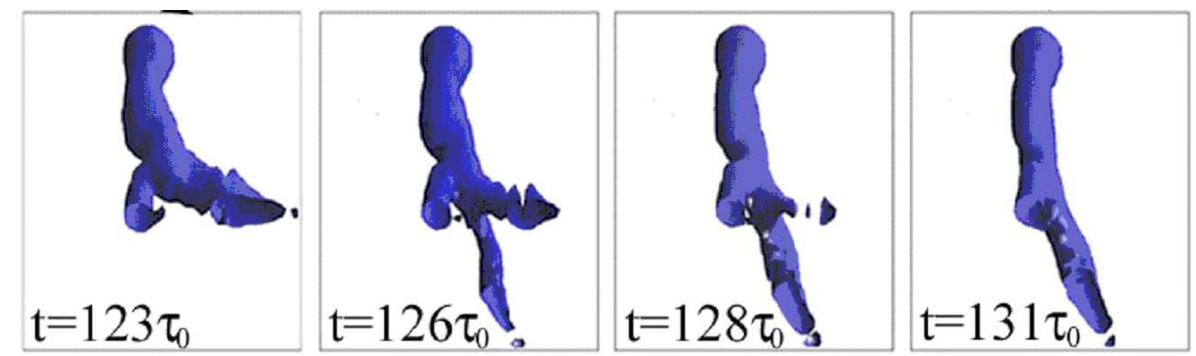

Fig. 2. Isosurface plots of a rotating structure presented in Fig. 1 at characteristic times.

For geometry and parameters that allow stable rotation, we investigate the existence of the solitonic $2 \mathrm{D}$ solutions. In order to do that, we consider Eqs. (1) and (2) in the steady state. Because of their symmetry, the above equations suggest the existence of a fundamental $2 \mathrm{D}$ vector soliton solution in the form

$$
F=u(x, y) \cos \theta \exp (\mathrm{i} \mu z), \quad B=u(x, y) \sin \theta \exp (-\mathrm{i} z \mu),
$$

where $\mu$ is the propagation constant and $\theta$ is an arbitrary projection angle $(\theta=\pi / 4$ here; the same analysis also corresponds to the copropagating geometry but with the choice $\theta=0$ ). When this solution is substituted into Eqs. (1), they both 
transform into one, degenerate equation

$$
-\mu u+\Delta u+\Gamma u \frac{|u|^{2}+I_{\mathrm{g}}}{1+|u|^{2}+I_{\mathrm{g}}}=0,
$$

The solitonic solutions can be found from Eq. (4) by using the modified Petviashvili iteration method. Here we just determine different classes of spatial vector solitons, assuming that they are stable and observable over certain crystal lengths.

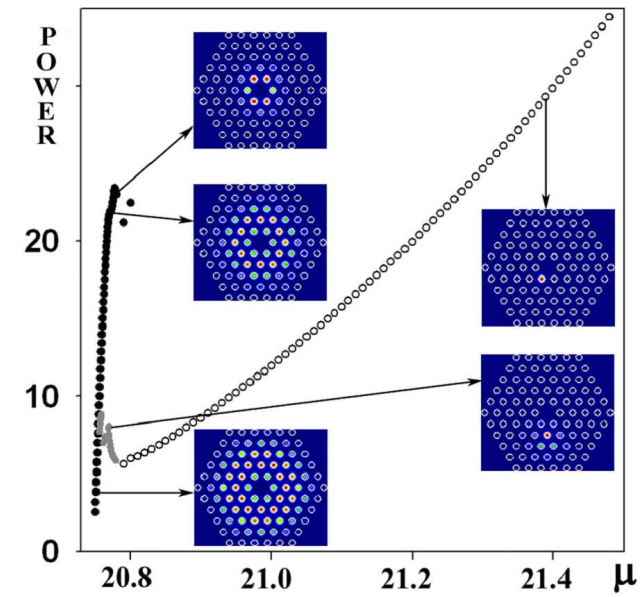

Fig. 3. Power diagram of solitonic solutions. Various symbols indicate different kinds of solitonic solution, characterized by corresponding profiles. Lattice parameters are as in Fig. 1 , and $\Gamma=25$.

Figure 3 shows power diagram together with the characteristic solitonic solutions. In the search for solitonic solutions, we used the same Gaussian input beams and parameters $\Gamma$ as in the numerical simulations. We varied the propagation constant $\mu$ to find the beam power $\left(P=\int_{-\infty}^{\infty} \int_{-\infty}^{\infty}|u|^{2} \mathrm{~d} x \mathrm{~d} y\right)$ corresponding to the beam power of the stable rotating structures. We found power for $\mu=20.751$ $(P=3.80)$ (shown by appropriate profiles in Fig. 3). Such solitonic solutions could be found only in the very narrow region near the lowest value of $\mu$. Of course, because of the $\mathrm{CP}$ geometry, these solitonic solutions are stable, but only up to the some critical values of propagation distance [21]. In Fig. 3 we present only those intervals of $\mu$, since there are no solitons for lower values of $\mu$, while for larger values solitonic solutions are nonphysical owing to the very large peak intensity (in comparison with the maximum lattice intensity $I_{0}$ ).

In power diagram (Fig. 3) filled circles represent all characteristic types of symmetric discrete solitonic solution. By increasing the propagation constant $\mu$ these solutions become more localized. Only for the beam powers corresponding to the less localized solitonic solutions one can find Gaussian-induced rotation in numerical simulations. Similar power diagrams are found for the same lattice 
geometry, but for different values of the coupling constant $\Gamma$. Again, the same types of symmetric discrete solitonic solution are found, and, for the beam powers corresponding to the less confined solitonic solutions, Gaussian-induced rotation can be found for some values of propagation distance. This procedure provides a more convenient way of finding Gaussian-induced rotation in numerics, as well as in experimental consideration of this interesting phenomenon.

\section{Conclusion}

In conclusion, we stress that, in contrast to the results given in the literature, where 2D spatial rotation in ring periodic lattices were discussed, we observed 3D time-dependent rotation in triangular PL. For the parameters of such rotation, we found solitonic solutions that correspond to the lowest values of the propagation constant in the power diagram. To find rotating structures for the discussed lattice geometry, it is more convenient to find less confined symmetric solitonic solutions by varying propagation constant $\mu$ and then, for the same coupling constant $\Gamma$ and beam power, to look for the propagating distance for which rotation occurs, either in numerical simulations or in experiments. Moreover, we believe that our procedure is applicable, besides to Kerr-type nonlinearities, to any system that can be described by a modified nonlinear Schrödinger equation with a periodic potential, such as Bose-Einstein condensates in optical lattices.

\section{Acknowledgments}

The authors acknowledge a financial support of the Ministry of Science of the Republic of Serbia (grant No. OI 141031).

\section{References}

[1] J.D. Joannopoulos, R.D. Meade, J.N. Winn, Photonic Crystals: Molding the Flow of Light, Princeton University Press, Princeton 1995.

[2] N.K. Efremidis, S. Sears, D.N. Christodoulides, J.W. Fleischer, M. Segev, Phys. Rev. E 66, 046602 (2002).

[3] D.N. Christodoulides, F. Lederer, Y. Silberberg, Nature 424, 817 (2003).

[4] J. Fleischer, T. Carmon, M. Segev, N. Efremidis, D. Christodoulides, Phys. Rev. Lett. 90, 023902 (2003).

[5] Y.S. Kivshar, G.P. Agrawal, Optical Solitons: from Fibers to Photonic Crystals, Academic, San Diego 2003.

[6] D.N. Christodoulides, R.I. Joseph, Opt. Lett. 13, 794 (1988).

[7] H. Eisenberg, Y. Silberberg, R. Morandotti, A. Boyd, J. Aitchison, Phys. Rev. Lett. 81, 3383 (1998).

[8] J.W. Fleischer, M. Segev, N.K. Efremidis, D.N. Christodoulides, Nature 422, 147 (2003).

[9] D.N. Neshev, T.J. Alexander, E.A. Ostrovskaya, Y.S. Kivshar, H. Martin, I. Makasyuk, Z. Chen, Phys. Rev. Lett. 92, 123903 (2004). 
[10] J.W. Fleischer, G. Bartal, O. Cohen, O. Manela, M. Segev, J. Hudock, D.N. Christodoulides, Phys. Rev. Lett. 92, 123904 (2004).

[11] Y.V. Kartashov, V.A. Vysloukh, L. Torner, Opt. Lett. 30, 637 (2005).

[12] X. Wang, Z. Chen, P.G. Kevrekidis, Phys. Rev. Lett. 96, 083904 (2006).

[13] B.B. Baizakov, B.A. Malomed, M. Salerno, Phys. Rev. E 74, 066615 (2006).

[14] M.S. Petrovic, Opt. Express 14, 9415 (2006).

[15] H. Trompeter, T. Pertsch, F. Lederer, D. Michaelis, U. Streppel, A. Bräuer, U. Peschel, Phys. Rev. Lett. 96, 023901 (2006).

[16] H. Trompeter, W. Krolikowski, D. Neshev, A. Desyatnikov, A. Sukhorukov, Y. Kivshar, T. Pertsch, U. Peschel, F. Lederer, Phys. Rev. Lett. 96, 053903 (2006).

[17] V.I. Petviashvili, Plasma Phys. 2, 469 (1976).

[18] J. Yang, I. Makasyuk, A. Bezryadina, Z. Chen, Stud. Appl. Math. 113, 389 (2004).

[19] M. Belic, D. Jovic, S. Prvanovic, D. Arsenovic, M. Petrovic, Opt. Express 14, 794 (2006).

[20] L. Larger, J.P. Goedgebuer, T. Erneux, Phys. Rev. E 69, 036210 (2004).

[21] K. Motzek, Ph. Jander, A. Desyatnikov, M. Belic, C. Denz, F. Kaiser, Phys. Rev. E 68, 066611 (2003). 